\title{
DATA DRIVEN SIMULATION WITH APPLICATIONS TO SYSTEM IDENTIFICATION
}

\author{
Ivan Markovsky *, Jan C. Willems *, Paolo Rapisarda ${ }^{* *}$ and \\ Bart L.M. De Moor* \\ * ESAT, SCD-SISTA, K.U.Leuven, Kasteelpark Arenberg 10, \\ B-3001 Leuven, Belgium \\ ${ }^{* *}$ Dept. Mathematics, University of Maastricht, 6200 MD \\ Maastricht, The Netherlands
}

\begin{abstract}
We present an iterative algorithm for computation of linear time-invariant system responses directly from an exact finite input/output trajectory of that system. In particular, the impulse response can be obtained from data, which opens the possibility to use realization theory in order to retrieve the system parameters. The oblique projection, used in subspace identification, computes free responses which initial conditions form a state sequence. We show that the oblique projection corresponds to a block version of the data driven simulation algorithm. The iterative algorithm, however, has computational advantages over the block algorithm, which makes it attractive for application in subspace algorithms. Copyright ${ }^{\oplus} 2005$ IFAC
\end{abstract}

Keywords: Deterministic identification, impulse response estimation, oblique projection.

\section{INTRODUCTION}

A precursor of the subspace identification methods is (Gopinath, 1969). In 1969 realization theory was well developed but it addresses a very special identification problem-the one starting from a given impulse response. So realization theory was not applicable at that time to the general deterministic identification problem and different approaches were searched. The first solution is proposed in (Gopinath, 1969) and is refined in (Budin, 1971; Liu and Suen, 1977). More recently, the deterministic identification problem is treated in the behavioral setting (Willems, 1986) and via the subspace methods (Van Overschee and De Moor, 1996, Chapter 2).

In this paper, we propose an algorithm that fulfills the missing link to realization theory. Therefore, we have a solution to the deterministic identification problem that could have been the first deterministic identification algorithm. Actually the algorithm that we propose is more general as it allows the computation of an ar- bitrary response directly from a given (possibly finite) trajectory of the system.

A trivial modification of the algorithm allows the computation of free responses of the system, which initial conditions form a valid state sequence. Such responses are called sequential. They are an essential ingredient of the N4SID type subspace identification methods and are computed by the oblique projection. We show that the oblique projection corresponds to a block version of the proposed algorithm and point out some advantages of the iterative algorithm.

The MOESP type algorithms use free responses that need not be sequential. The computational tool in this case is the orthogonal projection. We show that the orthogonal projection can also be inferred from the basic idea of this paper: computation of a response of the system directly from data. 


\section{DATA DRIVEN SIMULATION}

Consider a discrete-time linear time-invariant (LTI) system $\Sigma$, induced by a minimal state space representation

$$
\sigma x=A x+B u, \quad y=C x+D u,
$$

where $x(t) \in \mathbb{R}^{\mathrm{n}}, u(t) \in \mathbb{R}^{\mathrm{m}}, y(t) \in \mathbb{R}^{\mathrm{p}}$, and $\sigma$ is the backward shift operator $(\sigma f)(t)=f(t+1)$. Let $\mathscr{B}_{T}$ be the set of all $T$ samples long trajectories $w:=(u, y)$ of $\Sigma$, i.e.,

$$
\begin{aligned}
\mathscr{B}_{T} & :=\left\{w:=(u, y) \in\left(\mathbb{R}^{\mathrm{m}}\right)^{T} \times\left(\mathbb{R}^{\mathrm{p}}\right)^{T} \mid\right. \\
\exists x & \left.\in\left(\mathbb{R}^{\mathrm{n}}\right)^{T} \text { s.t. } \sigma x=A x+B u, y=C x+D u\right\} .
\end{aligned}
$$

The system $\Sigma$ is controllable if for any two trajectories $w_{1}, w_{2} \in \mathscr{B}:=\mathscr{B}_{\infty}$, there is a third trajectory $w \in \mathscr{B}$ and a natural number $t^{\prime} \in \mathbb{N}$, such that $w_{1}(t)=w(t)$, for all $t<0$, and $w_{2}(t)=w(t)$, for all $t \geq t^{\prime}$, see (Willems, 1991, Section 5). The usual definition of state controllability, given in terms of a state space representation of the system $\Sigma$, is equivalent to the above definition, given in terms of the behavior $\mathscr{B}$.

The lag 1 of the system $\Sigma$ is defined as its observability index, i.e., the smallest integer $i$, such that

$$
\operatorname{col}\left(C, C A, \ldots, C A^{i-1}\right)
$$

has full column rank. Equivalently, 1 is the minimal degree of a difference equation representation of the system $\Sigma$. Generically, $1=\lceil\mathrm{n} / \mathrm{p}\rceil$, where $\lceil r\rceil, r \in \mathbb{R}$, denotes the smallest integer larger than or equal to $r$. We will need often the following fact: if

$$
w_{1}:=\left(\left[\begin{array}{c}
u_{\mathrm{p}} \\
u_{\mathrm{f}}
\end{array}\right],\left[\begin{array}{c}
y_{\mathrm{p}} \\
y_{\mathrm{f}, 1}
\end{array}\right]\right) \in \mathscr{B}_{T}
$$

and

$$
w_{2}:=\left(\left[\begin{array}{l}
u_{\mathrm{p}} \\
u_{\mathrm{f}}
\end{array}\right],\left[\begin{array}{c}
y_{\mathrm{p}} \\
y_{\mathrm{f}, 2}
\end{array}\right]\right) \in \mathscr{B}_{T},
$$

where $\left(u_{\mathrm{p}}, y_{\mathrm{p}}\right)$ is at least 1 samples long, then $y_{\mathrm{f}, 1}=$ $y_{\mathrm{f}, 2}$, i.e., matching the first 1 samples of the trajectories $w_{1}$ and $w_{2}$, ensures that the initial conditions for the responses $y_{\mathrm{f}, 1}$ and $y_{\mathrm{f}, 2}$ are equal.

Throughout the paper, n, m, p, l, and $\mathscr{B}$ are fixed symbols denoting respectively the minimal order, the number of inputs and outputs, the lag, and the behavior of the system $\Sigma$. The considered problem is defined as follows.

Problem 1. (Data driven simulation). Given a trajectory $(\tilde{u}, \tilde{y}) \in \mathscr{B}_{T}$ of an LTI system $\Sigma$, an upper bound $\mathrm{n}_{\max }$ of the order $\mathrm{n}$, an upper bound $\mathrm{l}_{\max }$ of the lag 1 , and a time series $u_{\mathrm{f}} \in\left(\mathbb{R}^{\mathrm{m}}\right)^{t}$, where $t \in \mathbb{N}$, find the response $y_{\mathrm{f}}$ of the system $\Sigma$ under zero initial conditions and input $u_{\mathrm{f}}$.

Problem 1 can be solved indirectly by first identifying the system $\Sigma$ from the given data $(\tilde{u}, \tilde{y}), \mathrm{n}_{\max }, 1_{\max }$ and then simulating the desired response $y_{\mathrm{f}}$. Our goal, however, is to find a direct solution that does not find as an intermediate step a (more structured) representation of the system $\Sigma$. Such an algorithm can be viewed as performing data driven simulation of the implicitly defined by the data system $\Sigma$.

Note 1. (Nonzero initial conditions). The restriction that the desired response should be a zero initial conditions response can be relaxed. A way to specify the desired initial condition, say $x_{\mathrm{f}}$, is to give a trajectory $\left(u_{\mathrm{p}}, y_{\mathrm{p}}\right)$ of the system that transfers it to the state $x_{\mathrm{f}}$. Such a trajectory always exists, provided the system $\Sigma$ is controllable. For example, one can take

$$
u_{\mathrm{p}}=\left[\begin{array}{c}
0_{1_{\max } \mathrm{m} \times 1} \\
\bar{u}_{\mathrm{p}}
\end{array}\right], \quad y_{\mathrm{p}}=\left[\begin{array}{c}
0_{1_{\max } \mathrm{p} \times 1} \\
\bar{y}_{\mathrm{p}}
\end{array}\right],
$$

where $\bar{u}_{\mathrm{p}}$ is an input that transfers $\Sigma$ from the zero state to the state $x_{\mathrm{f}}$ and $\bar{y}_{\mathrm{p}}$ is the corresponding output. In Note 2 we explain how $\left(u_{\mathrm{p}}, y_{\mathrm{p}}\right)$ can be used for simulation of non-zero initial condition responses.

Let $\mathscr{H}_{\Delta}(\bullet)$ be a block-Hankel matrix with $\Delta$ blockrows, e.g., with $\tilde{u}=(\tilde{u}(1), \ldots, \tilde{u}(T))$,

$$
\mathscr{H}_{\Delta}(\tilde{u})=\left[\begin{array}{cccc}
\tilde{u}(1) & \tilde{u}(2) & \cdots & \tilde{u}(T-\Delta+1) \\
\tilde{u}(2) & \tilde{u}(3) & \cdots & \tilde{u}(T-\Delta+2) \\
\vdots & \vdots & & \vdots \\
\tilde{u}(\Delta) & \tilde{u}(\Delta+1) & \cdots & \tilde{u}(T)
\end{array}\right] .
$$

A time series $\tilde{u}$ is called persistently exciting of or$\operatorname{der} \Delta$ if the matrix $\mathscr{H}_{\Delta}(\tilde{u})$ is of full row rank.

The solution of Problem 1 is based on the following lemma.

\section{Lemma 2. ((Willems et al., 2004)). Assume that}

1. the LTI system $\Sigma$ is controllable,

2. $\tilde{u}$ is persistently exciting of order $\Delta+\mathrm{n}$, where $\Delta \in \mathbb{N}$ and $n$ is the order of $\Sigma$, and

3. $(\tilde{u}, \tilde{y})$ is a trajectory of $\Sigma$, i.e., $(\tilde{u}, \tilde{y}) \in \mathscr{B}_{T}$, for certain $T \in \mathbb{N}$.

Then

$$
\operatorname{image}\left(\left[\begin{array}{c}
\mathscr{H}_{\Delta}(\tilde{u}) \\
\mathscr{H}_{\Delta}(\tilde{y})
\end{array}\right]\right)=\mathscr{B}_{\Delta} \text {. }
$$

Assumption 1 is a part of the problem formulation and is a standing assumption throughout the paper. Admittedly, it is most restrictive for practical applications but in the same time it is a convenient one for theoretical studies. Realization theory relies on the stronger assumption that the exact impulse response of the system is given. Also classical identification theory starts from the similar assumption that the given data is generated by a stochastic ARMAX or ARMA system. Assumption 3 is mild in the sense that the set of controllable systems is generic in the set of all systems. Assumption 2 is nontrivial and as we will show in Sections 3 and 4, it gives an answer to an open question in subspace system identification: what is the sharpest verifiable from the given data $(\tilde{u}, \tilde{y})$ condition for identifiability of the system $\Sigma$. 
Lemma 2 shows that if $\tilde{u}$ is persistently exciting of sufficient order, any $\Delta$ samples long trajectory of the system is of the form $\left[\begin{array}{l}\mathscr{H}_{\Delta}(\tilde{u}) \\ \mathscr{H}_{\Delta}(\tilde{y})\end{array}\right] g$, for some $g \in \mathbb{R}^{j}$, where $j:=T-\Delta+1$. Now take $\Delta=t$, the length of the desired response $y_{\mathrm{f}}$. In Theorem 3 , we specify how a $g$ that corresponds to the trajectory $\left(u_{\mathrm{f}}, y_{\mathrm{f}}\right)$ can be found.

Theorem 3. Let $\Sigma$ be controllable, $(\tilde{u}, \tilde{y}) \in \Sigma$, and $\tilde{u}$ be persistently exciting of order $\Delta+1_{\max }+\mathrm{n}_{\max }$. Define the matrices $U_{\mathrm{p}} \in \mathbb{R}^{1_{\max } \mathrm{m} \times j}, U_{\mathrm{f}} \in \mathbb{R}^{\Delta \mathrm{m} \times j}, Y_{\mathrm{p}} \in \mathbb{R}^{1_{\max } \times j}$, $Y_{\mathrm{f}} \in \mathbb{R}^{\Delta \mathrm{p} \times j}$, where $j:=T-\Delta-1_{\max }+1$, by

$$
\mathscr{H}_{1_{\max }+\Delta}(\tilde{u})=:\left[\begin{array}{c}
U_{\mathrm{p}} \\
U_{\mathrm{f}}
\end{array}\right], \quad \mathscr{H}_{1_{\max }+\Delta}(\tilde{y})=:\left[\begin{array}{c}
Y_{\mathrm{p}} \\
Y_{\mathrm{f}}
\end{array}\right] .
$$

Then the system of equations

$$
\left[\begin{array}{c}
U_{\mathrm{p}} \\
U_{\mathrm{f}} \\
Y_{\mathrm{p}}
\end{array}\right] g=\left[\begin{array}{c}
0 \\
u_{\mathrm{f}} \\
0
\end{array}\right]
$$

is solvable for any $u_{\mathrm{f}}$ and any particular solution $\bar{g}$ allows the computation of the response $y_{\mathrm{f}}$ of $\Sigma$ due to the input $u_{\mathrm{f}}$ and zero initial conditions as $y_{\mathrm{f}}=Y_{\mathrm{f}} \bar{g}$.

PROOF. The statement is proven for the special case of an impulse response in (Markovsky et al., 2004, Section 3). Here we adapt the proof for the general case.

Under the assumptions of the theorem, we can apply Lemma 2 from which follows that

$$
\operatorname{image}\left(\left[\begin{array}{l}
\mathscr{H}_{1_{\max }+\Delta}(\tilde{u}) \\
\mathscr{H}_{1_{\max }+\Delta}(\tilde{y})
\end{array}\right]\right)=\mathscr{B}_{1_{\max }+\Delta} .
$$

The time series $\left(u_{\mathrm{f}}, y_{\mathrm{f}}\right)$ is a zero initial conditions trajectory of $\Sigma$, so that preceded by any number of zeros, it remains a trajectory of $\Sigma$, i.e.,

$$
\left(\left[\begin{array}{c}
0_{1_{\max } \mathrm{m} \times 1} \\
u_{\mathrm{f}}
\end{array}\right],\left[\begin{array}{c}
0_{1_{\max } \mathrm{p} \times 1} \\
y_{\mathrm{f}}
\end{array}\right]\right) \in \mathscr{B}_{1_{\max }+\Delta} .
$$

Therefore, there exists a vector $\bar{g}$, such that

$$
\left[\begin{array}{c}
U_{\mathrm{p}} \\
U_{\mathrm{f}} \\
Y_{\mathrm{p}} \\
Y_{\mathrm{f}}
\end{array}\right] \bar{g}=\left[\begin{array}{c}
0_{1_{\max } \mathrm{m} \times 1} \\
u_{\mathrm{f}} \\
0_{1_{\max } \times 1} \\
y_{\mathrm{f}}
\end{array}\right] .
$$

This shows that there exists a solution $\bar{g}$ of (2) and therefore $Y_{\mathrm{f}} \bar{g}$ is the desired response.

Conversely, let $g$ be a solution of (2). We have

$$
\left[\begin{array}{c}
U_{\mathrm{p}} \\
U_{\mathrm{f}} \\
Y_{\mathrm{p}} \\
Y_{\mathrm{f}}
\end{array}\right] g=\left[\begin{array}{c}
0_{1_{\max } \mathrm{m} \times 1} \\
u_{\mathrm{f}} \\
0_{1_{\max } \mathrm{p} \times 1} \\
Y_{\mathrm{f}} g
\end{array}\right]
$$

and by Lemma 2

$$
\left(\left[\begin{array}{c}
0_{1_{\max } \mathrm{m} \times 1} \\
u_{\mathrm{f}}
\end{array}\right],\left[\begin{array}{c}
0_{1_{\max } \mathrm{p} \times 1} \\
Y_{\mathrm{f}} g
\end{array}\right]\right) \in \mathscr{B}_{1_{\max }+l} .
$$

This trajectory is identically zero during the first $l_{\max }$ samples, which (using the assumption $1_{\max } \geq 1$ ) guarantees that the initial conditions are set to zero. Then $Y_{\mathrm{f}} g$ is indeed $y_{\mathrm{f}}$.
Note 2. (Splitting of the data into "past" and "future"). The block-Hankel matrices $\mathscr{H}_{1_{\max }+\Delta}(\tilde{u}), \mathscr{H}_{1_{\max }+\Delta}(\tilde{y})$ are split into two parts, see (1). We refer to the blocks $U_{\mathrm{p}}$ and $Y_{\mathrm{p}}$ as the "past" and to the blocks $U_{\mathrm{f}}$ and $Y_{\mathrm{f}}$ as the "future". Let $\operatorname{row} \operatorname{dim}(\bullet)$ denotes the number of block row of its argument. We have that row $\operatorname{dim}\left(U_{\mathrm{p}}\right)=$ row $\operatorname{dim}\left(Y_{\mathrm{p}}\right)=1_{\text {max }}$ and row $\operatorname{dim}\left(U_{\mathrm{f}}\right)=$ row $\operatorname{dim}\left(Y_{\mathrm{f}}\right)=\Delta$. The reason for this partitioning is to fix the initial conditions for the computed response in the "future". The "past" is sufficiently long to set the correct initial condition and the "future" is used for computation of a response, starting from this initial condition. In Theorem 3 we fix zero initial conditions by assigning the "past" to zero. Assigning the "past" to the trajectory $\left(u_{\mathrm{p}}, y_{\mathrm{p}}\right)$, defined in Note 1 , allows to compute a nonzero initial conditions response.

Theorem 3 readily gives an algorithm (see Algorithm 1) for the computation of the response $y_{\mathrm{f}}$. A hidden limitation of Algorithm 1, however, is that the persistency of excitation condition requires a sufficiently large length $T$ of given time series $(\tilde{u}, \tilde{y})$. Finite, $T$ samples long data sequence $(\tilde{u}, \tilde{y})$ implies that the length $t=\Delta$ of the response $y_{\mathrm{f}}$ that can be computed by the block algorithm is

$$
\Delta \leq \frac{T+1}{\mathrm{~m}+1}-\mathrm{I}_{\max }-\mathrm{n}_{\max } .
$$

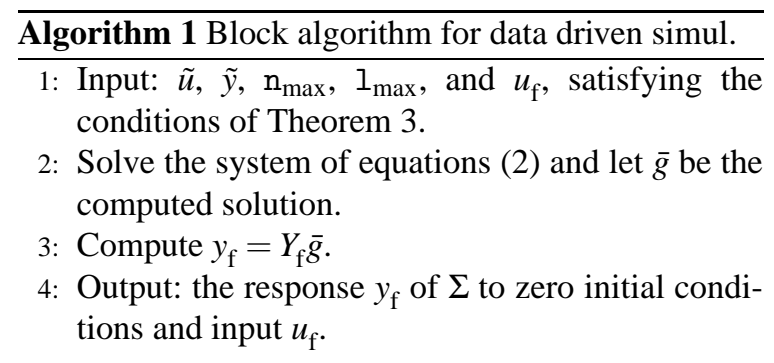

It is possible, however, to find an arbitrary long response from a finite amount of data by computing iteratively $\Delta$ samples long responses $y_{\mathrm{f}}^{(0)}, y_{\mathrm{f}}^{(1)}, \ldots$, where $\Delta$ satisfies (4). These blocks are made sequential pieces of the desired response $y_{\mathrm{f}}$ by matching their initial conditions. The iterative version of Algorithm 1 (see Algorithm 2) is based on the following lemma.

Lemma 4. ((Markovsky et al., 2004)). Let $\tilde{w}_{1} \in \mathscr{B}_{T_{1}}$, $\tilde{w}_{2} \in \mathscr{B}_{T_{2}}$, and let the last $l_{\max }$ samples of $\tilde{w}_{1}$ coincide with the first $l_{\max }$ samples of $\tilde{w}_{2}$, where $l_{\max }$ is larger than or equal to the lag of $\Sigma$. Then

$$
\begin{aligned}
w:= & \left(\tilde{w}_{1}(1), \ldots, \tilde{w}_{1}\left(T_{1}\right),\right. \\
& \left.\tilde{w}_{2}\left(1_{\max }+1\right), \ldots, \tilde{w}_{2}\left(T_{2}\right)\right) \in \mathscr{B}_{T_{1}+T_{2}-1_{\max }} .
\end{aligned}
$$

In the outline of Algorithm 2, we use the notation $f\left(t_{1}: t_{2}\right):=\operatorname{col}\left(f\left(t_{1}\right), \ldots, f\left(t_{2}\right)\right)$. Acting on a block matrix, $\sigma$ removes its first block row.

Note 3. (About the choice of $\Delta$ ). Let $\tilde{u}$ be persistently exciting of order $i$. Algorithm 2 can be applied if 


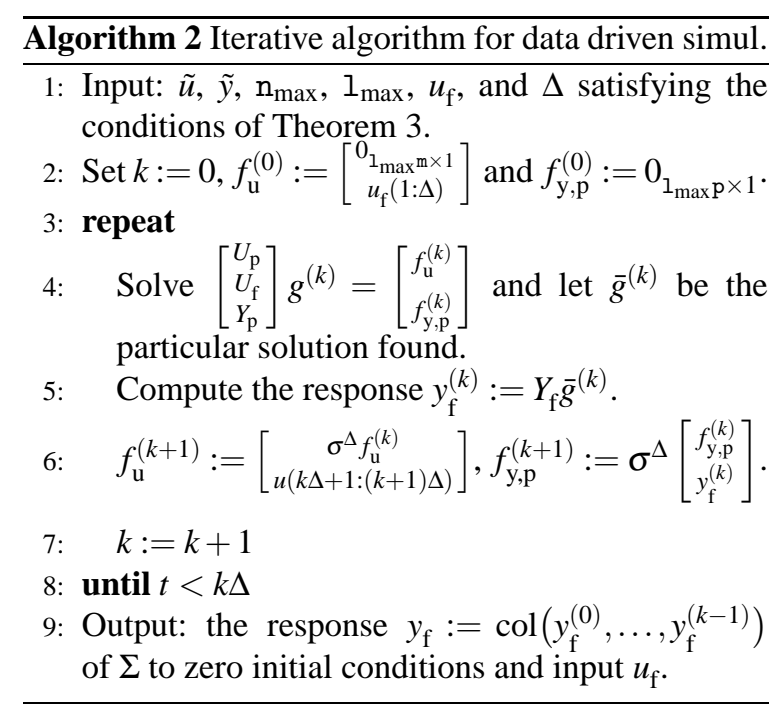

$i>\mathrm{n}_{\max }+\mathrm{l}_{\max }$, in which case the parameter $\Delta$ can be chosen in the interval $\left[1, i-\mathrm{n}_{\max }-1_{\max }\right]$. The choice of $\Delta$ affects the performance of the algorithm: the amount of computations is a function of $\Delta$ and, in the presence of noise on the data $(\tilde{u}, \tilde{y})$, the accuracy of the computed result is a function of $\Delta$. These or other criteria can be optimized by selecting a suitable $\Delta$. The freedom to choose $\Delta$ is an important advantage of Algorithm 2 over Algorithm 1.

Algorithm 1 is a special case of Algorithm 2. Indeed by taking $\Delta=t$, Algorithm 2 reduces to the block computation. There are a number of advantages in doing the computations iteratively, however. Most importantly, for large $t$, Algorithm 1 might not be applicable, while Algorithm 2 still be. Algorithm 1 assumes persistency of excitation of order proportional to $t$. By using the idea of weaving responses as stated in Lemma 4, we make the persistency of excitation condition independent of $t$. With $\Delta=1$ (the minimal possible), the assumptions of Algorithm 2 imply $T \geq$ $\mathrm{m}\left(\mathrm{l}_{\max }+\mathrm{n}_{\max }+1\right)+\mathrm{l}_{\max }+\mathrm{n}_{\max }$, which is a function of $\mathrm{n}_{\max }, \mathrm{l}_{\max }$, and $\mathrm{m}$ only. As shown in (Willems et $a l ., 2004)$, this is a necessary condition for identifiability of $\Sigma$.

Even when Algorithm 1 is applicable, from an efficiency point of view it might not be optimal. The freedom to choose the parameter $\Delta$, as discussed in Note 3, can be used to optimize the efficiency of the computations. In addition, in the presence of noise on the data $(\tilde{u}, \tilde{y})$, the accuracy of Algorithm 2 depends on the choice of $\Delta$ and a proper choice of $\Delta$ makes Algorithm 2 superior to Algorithm 1.

In the next two sections, we show that data driven simulation is a hidden core subproblem of the subspace identification algorithms.

\section{COMPUTATION OF FREE RESPONSES}

An essential step of the MOESP algorithms is the orthogonal projection of the rows of $\mathscr{H}_{\mathrm{n}_{\max }}(\tilde{y})$ on the orthogonal complement of the row space of $\mathscr{H}_{\mathrm{n}_{\max }}(\tilde{u})$, i.e., the MOESP algorithms compute the matrix

$$
Y_{0}:=\mathscr{H}_{\mathrm{n}_{\max }}(\tilde{y}) \Pi_{\tilde{u}}^{\perp} .
$$

where

$\Pi_{\tilde{u}}^{\perp}:=I-\mathscr{H}_{\mathrm{n}_{\max }}^{\top}(\tilde{u})\left(\mathscr{H}_{\mathrm{n}_{\max }}(\tilde{u}) \mathscr{H}_{\mathrm{n}_{\max }}^{\top}(\tilde{u})\right)^{-1} \mathscr{H}_{\mathrm{n}_{\max }}(\tilde{u})$.

(For well posedness of this operation $\tilde{u}$ is assumed to be persistently exciting of order $\mathrm{n}_{\max }$.) In subspace identification it is customary to think in terms of geometric operations: projection of the rows of a certain matrix onto the row space of another matrix. The fact that these matrices have special (block-Hankel) structure is ignored and the link with systems theory is lost. Still, as we show next, the orthogonal projection (5) has a simple and useful system theoretic interpretation.

Observe that

$$
\left[\begin{array}{l}
\mathscr{H}_{\mathrm{n}_{\max }}(\tilde{u}) \\
\mathscr{H}_{\mathrm{n}_{\max }}(\tilde{y})
\end{array}\right] \Pi_{\tilde{u}}^{\perp}=\left[\begin{array}{c}
0 \\
Y_{0}
\end{array}\right] .
$$

Since by assumption the columns of $\left[\begin{array}{l}\mathscr{H}_{\max }(\tilde{u}) \\ \mathscr{H}_{\mathrm{n}_{\max }}(\tilde{y})\end{array}\right]$ are trajectories of $\Sigma$, it follows by the linearity of $\Sigma$ that the columns of $\left[\begin{array}{c}0 \\ Y_{0}\end{array}\right]$ are also trajectories of $\Sigma$. The inputs of the derived trajectories are identically zero, so that the columns of $Y_{0}$ are free responses of $\Sigma$. Therefore, $Y_{0}$ must be of the form

$$
Y_{0}=\Gamma X_{0}, \text { where } \begin{aligned}
& X_{0} \in \mathbb{R}^{\mathrm{n} \times\left(T-\mathrm{n}_{\max }+1\right)} \text { and } \\
& \Gamma=\operatorname{col}\left(C, C A, C A^{2}, \ldots, C A^{\mathrm{n}_{\max }-1}\right)
\end{aligned}
$$

Assuming that $\operatorname{rank}\left(Y_{0}\right)=\mathrm{n}$ or equivalently that $\operatorname{rank}\left(X_{0}\right)=\mathrm{n}$, the parameters $A$ and $C$ can be computed (up to a similarity transformation) from $Y_{0}$. A way to do this is to compute a rank revealing factorization of $Y_{0}=L R$ and to solve the shift equation

$$
\left(\sigma^{*} L\right) A=(\sigma L),
$$

( $\sigma^{*} L$ is the matrix obtained from $L$ by removing its first block row) which is a well known technique from realization theory. In (Verhaegen and Dewilde, 1992, Sec. 3.3), it is proven that a sufficient condition for $\operatorname{rank}\left(Y_{0}\right)=\mathrm{n}$ is

$$
\operatorname{rank}\left(\left[\begin{array}{c}
X_{0} \\
\mathscr{H}_{\mathrm{n}_{\max }}(\tilde{u})
\end{array}\right]\right)=\mathrm{n}+\mathrm{n}_{\max } \mathrm{m} .
$$

This condition, however, is not verifiable from the data $(\tilde{u}, \tilde{y})$, either. Therefore, given $(\tilde{u}, \tilde{y})$, except for special cases ( $\tilde{u}$ white noise or periodic), one can not say whether the system $\Sigma$ is identifiable by the MOESP algorithms.

Next we show how the orthogonal projection (5) can be derived from the point of view of Lemma 2, i.e., from a system theoretic reasoning. Under the conditions of Lemma 2, there is a matrix $G$, such that

$$
\left[\begin{array}{c}
\mathscr{H}_{\mathrm{n}_{\max }}(\tilde{u}) \\
\mathscr{H}_{\mathrm{n}_{\max }}(\tilde{y})
\end{array}\right] G=\left[\begin{array}{c}
0 \\
Y_{0}
\end{array}\right] .
$$

Moreover, if $G$ has a maximal rank, then $\operatorname{rank}\left(Y_{0}\right)=$ $\mathrm{n}$. The projector $\Pi_{\tilde{u}}^{\perp}$ satisfies (7) and has the maximal rank $T-\mathrm{n}_{\max }(\mathrm{m}+1)+1$ among all matrices $G$ 
that satisfy (7). Therefore, $\mathscr{H}_{\mathrm{n}_{\max }}(\tilde{y}) \Pi_{\tilde{u}}^{\perp}=Y_{0}$ with $\operatorname{rank}\left(Y_{0}\right)=\mathrm{n}$ as desired. The assumptions of Lemma 2 requires that

$\tilde{u}$ is persistently exciting of order $\mathrm{n}_{\max }+\mathrm{n}$.

In (Willems et al., 2004, Corollary 1), it is proven that (8) implies assumption (6), so that (8) is a verifiable from the given data condition under which the system $\Sigma$ can be identified by the MOESP algorithms. In an appropriate sense, see (Willems et al., 2004), the condition is also necessary, i.e., it is sharp.

Note that the orthogonal projection (5) computes $j=$ $T-\mathrm{n}_{\max }+1$ free responses, where typically $j \gg \mathrm{n}_{\max }$. Actually for the computation of $A$ and $C$, n linearly independent $l_{\max }$ samples long free responses are needed. Since $\mathrm{n}$ is unknown, $\mathrm{n}_{\max }$ such responses should be computed. The obtained matrix $Y_{0}$ then corresponds to a non-minimal state space representation: $A \in \mathbb{R}^{\mathrm{n}_{\max } \times \mathrm{n}_{\max }}$ and $C \in \mathbb{R}^{\mathrm{p} \times \mathrm{n}_{\max }}$. In order to make it minimal, $\mathrm{n}$ linearly independent columns of $Y_{0}$ are selected and $Y_{0}$ is redefined to be the full column rank matrix of the selected columns. The SVD factorization, used in the MOESP algorithms does namely this step of making the non-minimal representation a minimal one.

The observation that the computation of $\mathrm{n}_{\max }$ instead of $j$ responses suffices results in a huge computational saving. Similar (but not equivalent) saving is achieved in the numerical implementation of the MOESP algorithms by what is known as the QR-trick. Let

$$
\left[\begin{array}{l}
\mathscr{H}_{\mathrm{n}_{\max }}(\tilde{u}) \\
\mathscr{H}_{\mathrm{n}_{\text {max }}}(\tilde{y})
\end{array}\right]^{\top}=Q R
$$

be the QR factorization of the matrix in the left hand side of the identity and partition $R$ as follows

$$
R^{\top}=:\left[\begin{array}{ccc}
\mathrm{n}_{\max } \mathrm{m} & \mathrm{n}_{\max } \mathrm{p} \\
R_{11} & 0 & 0 \\
R_{21} & R_{22} & 0
\end{array}\right] \mathrm{n}_{\max } \mathrm{p} .
$$

It can be shown, see (Verhaegen and Dewilde, 1992, Section 4.1), that

$$
\operatorname{image}\left(Y_{0}\right)=\operatorname{image}\left(R_{22}\right),
$$

so that only the $R_{22}$ block of the $R$ factor is needed for the computation of the parameters $A$ and $C$. Note that the QR-trick is a numerical linear algebra technique that is not motivated from the system theoretic considerations that we promote here.

\section{COMPUTATION OF SEQUENTIAL FREE RESPONSES}

Consider again the splitting of the data into "past" and "future" as defined in (1) with $\Delta=1_{\max }$ and let

$$
W_{\mathrm{p}}:=\left[\begin{array}{c}
U_{\mathrm{p}} \\
Y_{\mathrm{p}}
\end{array}\right] .
$$

As the key computational step of the MOESP algorithms is the orthogonal projection, the key computational step of the N4SID algorithms is the oblique projection of $Y_{\mathrm{f}}$ along the space spanned by the rows of $U_{\mathrm{f}}$ onto the space spanned by the rows of $W_{\mathrm{p}}$. This geometric operation, denoted by $Y_{\mathrm{f}} / U_{\mathrm{f}} W_{\mathrm{p}}$, is defined as follows, see (Van Overschee and De Moor, 1996, eqn. (1.4) on page 21):

$$
Y_{0}:=Y_{\mathrm{f}} /{ }_{U_{\mathrm{f}}} W_{\mathrm{p}}:=Y_{\mathrm{f}} \Pi_{\mathrm{obl}},
$$

where

$$
\Pi_{\mathrm{obl}}:=\left[\begin{array}{ll}
W_{\mathrm{p}}^{\top} & U_{\mathrm{f}}^{\top}
\end{array}\right]\left[\begin{array}{ll}
W_{\mathrm{p}} W_{\mathrm{p}}^{\top} & W_{\mathrm{p}} U_{\mathrm{f}}^{\top} \\
U_{\mathrm{f}} W_{\mathrm{p}}^{\top} & U_{\mathrm{f}} U_{\mathrm{f}}^{\top}
\end{array}\right]^{+}\left[\begin{array}{c}
W_{\mathrm{p}} \\
0
\end{array}\right] .
$$

As the orthogonal projection, the oblique projection also has a simple and useful system theoretic interpretation that is not clarified in the literature. Next we show that the oblique projection computes sequential free responses of the system.

Note that

$$
\left[\begin{array}{c}
W_{\mathrm{p}} \\
U_{\mathrm{f}} \\
Y_{\mathrm{f}}
\end{array}\right] \Pi_{\mathrm{obl}}=\left[\begin{array}{c}
W_{\mathrm{p}} \\
0 \\
Y_{0}
\end{array}\right]
$$

The first two block rows of the identity are easy to verify and the third one is true by definition. The columns of $\left[\begin{array}{l}W_{\mathrm{p}} \\ U_{\mathrm{f}} \\ Y_{\mathrm{f}}\end{array}\right]$ are trajectories of the system $\Sigma$ and by the linearity of $\Sigma$, the columns of $\left[\begin{array}{c}W_{\mathrm{p}} \\ 0 \\ Y_{0}\end{array}\right]$ are also trajectories of $\Sigma$. The derived from the data trajectories are such that the inputs are identically zero in the "future" (the part labeled with subscript f). Therefore, the corresponding responses $Y_{0}$ are free responses. Their initial conditions are set by the "past" (the part labeled with subscript p) and due to the Hankel structure of $U_{\mathrm{p}}$ and $Y_{\mathrm{p}}$, they are sequential. Therefore, $Y_{0}$ must be of the form

$Y_{0}=\Gamma X_{0}$, where $\begin{gathered}\Gamma=\operatorname{col}\left(C, C A, C A^{2}, \ldots, C A^{\mathrm{n}_{\max }-1}\right) \\ X_{0}=[x(1) \cdots x(j)]\end{gathered}$

and $x(1), \ldots, x(j)$ is a state sequence of $\Sigma$, with $j:=$ $T-2 \mathrm{n}_{\max }+1$.

Under the condition $\operatorname{rank}\left(X_{0}\right)=\mathrm{n}$, a state sequence, say $\tilde{x}(1), \ldots, \tilde{x}(j)$, of the system can be obtained from a rank revealing factorization of $Y_{0}$. Once a state sequence is available, the system parameters $(A, B, C, D)$ can be computed (in a basis of the state space, fixed by the rank revealing factorization) by solving the linear system of equations

$$
\left[\begin{array}{ccc}
\tilde{x}(2) & \cdots & \tilde{x}(j) \\
\tilde{y}(1) & \cdots & \tilde{y}(j-1)
\end{array}\right]=\left[\begin{array}{ll}
A & B \\
C & D
\end{array}\right]\left[\begin{array}{ccc}
\tilde{x}(1) & \cdots & \tilde{x}(j-1) \\
\tilde{u}(1) & \cdots & \tilde{u}(j-1)
\end{array}\right] .
$$

In (Van Overschee and De Moor, 1996, Section 2, Theorem 2), it is proven that a sufficient condition for $\operatorname{rank}\left(X_{0}\right)=\mathrm{n}$ is

1. $\tilde{u}$ persistently exciting of order $2 \mathrm{n}_{\max }$ and

2. row $\operatorname{span}\left(X_{0}\right) \cap$ row $\operatorname{span}\left(U_{\mathrm{f}}\right)=\{0\}$,

see assumption 1 and 2 of (Van Overschee and De Moor, 1996, Sec. 2, Thm 2). As with assumption (6) 
in the MOESP algorithms, however, assumption 2 is again not verifiable from the given data.

Next we show that the oblique projection (9) is an implementation of Algorithm 1 for the computation of sequential free responses. Under the assumptions of Lemma 2, a matrix $Y_{0}$ which columns are sequential free responses can be computed from the data by solving the system

$$
\left[\begin{array}{c}
W_{\mathrm{p}} \\
U_{\mathrm{f}}
\end{array}\right] G=\left[\begin{array}{c}
W_{\mathrm{p}} \\
0
\end{array}\right]
$$

and setting $Y_{0}=Y_{\mathrm{f}} G$. The zero block in the right hand side of (11) implies that the columns of $Y_{0}$ are free responses of the system and the Hankel structure of $U_{\mathrm{p}}$ and $Y_{\mathrm{p}}$ implies that the columns of $Y_{0}$ are sequential. The projector $\Pi_{\mathrm{obl}}$ is the least squares least norm solution of (11), so that $\Pi_{\mathrm{obl}}$ is a particular solution of (11). Then the oblique projection $Y_{\mathrm{f}} \Pi_{\mathrm{obl}}$ is a matrix of sequential free responses, as desired. System (11) and $Y_{0}=Y_{\mathrm{f}} G$ correspond to Algorithm 1. Persistency of excitation of $\tilde{u}$ of order $2 \mathrm{n}_{\max }+\mathrm{n}$ (i.e., the assumption of Theorem 3 ) is a sufficient, verifiable from the data $(\tilde{u}, \tilde{y})$, condition for assumptions 1 and 2 of (Van Overschee and De Moor, 1996, Sec. 2, Thm 2).

The oblique projection like the orthogonal projection computes more responses than needed for the identification of the system. The matrix $Y_{0}$ computed via (9) is with $j=T-2 \mathrm{n}_{\max }+1$ columns and typically $j \gg \mathrm{n}_{\max }$. In fact, only $j=\mathrm{n}_{\max }+\mathrm{m}+2$ sequential free responses are enough for the identification of $\Sigma$. This follows from the fact that the system parameters $(A, B, C, D)$ have $(\mathrm{n}+\mathrm{m})(\mathrm{n}+\mathrm{p})$ unknowns, and for existence of solution of the system of equations (10), at least that many equations are needed. The numerical implementation of the N4SID algorithms uses the QR trick in order to achieve similar computational saving.

\section{CONCLUSIONS}

We have presented two algorithms for data driven simulation. The first one does the computation block-wise and the second one is iterative. A fundamental limitation of the block algorithm is that a persistency of excitation, proportional to the length of the computed response is assumed. This limitation is avoided by the iterative algorithm. In addition, the iterative algorithm has a tunable parameter - the length of a block computed at a time - that can be used to optimize the numerical efficiency or another criterion.

We showed that data driven simulation is relevant for deterministic system identification. From one side, the algorithms of this paper allow the computation of the impulse response directly from data and thus in combination with realization methods they provide a state space identification procedure. From another side, data driven simulation is a hidden core subproblem of the existing subspace identification methods: the orthogonal projection computes free responses and the oblique projection computes sequential free responses of the system. Moreover, the oblique projection corresponds to our block algorithm. We explained that the QR-trick in the subspace identification algorithm has the system theoretic significance of reducing the number of computed responses from data. Using the result of (Willems et al., 2004), we gave sufficient verifiable from the data conditions for identifiability.

\section{ACKNOWLEDGEMENTS}

Our research is supported by: Research Council KUL: GOAMefisto 666, several PhD/postdoc \& fellow grants; Flemish Government: FWO: PhD/postdoc grants, projects, G.0240.99 (multilinear algebra), G.0407.02 (support vector machines), G.0197.02 (power islands), G.0141.03 (Identification and cryptography), G.0491.03 (control for intensive care glycemia), G.0120.03 (QIT), G.0800.01 (collective intelligence), research communities (ICCoS, ANMMM); AWI: Bil. Int. Collaboration Hungary/ Poland; IWT: PhD Grants, Soft4s (softsensors), Belgian Federal Government: DWTC (IUAP IV-02 (1996-2001) and IUAP V-22 (2002-2006), PODO-II (CP/40: TMS and Sustainability); EU: CAGE; ERNSI; Eureka 2063IMPACT; Eureka 2419-FliTE; Contract Research/agreements: Data4s, Electrabel, Elia, LMS, IPCOS, VIB.

\section{REFERENCES}

Budin, M. A. (1971). Minimal realization of discrete linear systems from input-output observations. IEEE Trans. on Aut. Control 16(5), 395-401.

Gopinath, B. (1969). On the identification of linear time-invariant systems from input-output data. The Bell System Technical J. 48(5), 1101-1113.

Liu, R. and L.-C. Suen (1977). Minimal dimension realization and identifiability of inputoutput sequences. IEEE Trans. on Aut. Control 22(2), 227-232.

Markovsky, I., J. C. Willems, P. Rapisarda and B. De Moor (2004). Algorithms for deterministic balanced subspace identification. Technical Report 04-13. Dept. EE, K.U. Leuven.

Van Overschee, P. and B. De Moor (1996). Subspace Identification for Linear Systems: Theory, Implementation, Applications. Kluwer Academic Publishers.

Verhaegen, M. and P. Dewilde (1992). Subspace model identification, Part I: The output error state space model identification class of algorithms. Int. J. Control 56, 1187-1210.

Willems, J. C. (1986). From time series to linear system-Part II. Exact modelling. Automatica 22(6), 675-694.

Willems, J. C. (1991). Paradigms and puzzles in the theory of dynamical systems. IEEE Trans. on Aut. Control 36(3), 259-294.

Willems, J. C., P. Rapisarda, I. Markovsky and B. De Moor (2004). A note on persistency of excitation. Systems \& Control Letters. 